\title{
COR TRIATRIATUM DEXTER
}

Debasish Das ${ }^{1}$, Satyabrata Guru ${ }^{2}$

\section{HOW TO CITE THIS ARTICLE:}

Debasish Das, Satyabrata Guru. "COR Triatriatum Dexter". Journal of Evolution of Medical and Dental Sciences 2015; Vol. 4, Issue 79, October 01; Page: 13904-13907, DOI: 10.14260/jemds/2015/1980

ABSTRACT: Cor triatriatum dexter is a rare congenital cardiac anomaly where the right atrium is divided into two chambers by a membrane. We report a 45 year old female who was having effort intolerance NYHA class II admitted in the gynecology ward of AIIMS, Bhubaneswar to undergo resection of an ovarian tumour and diagnosed to have cor triatriatum dexter by transthoracic echocardiography and the membrane was non-obstructive The pathophysiology, approach to the diagnosis, and mode of treatment are also discussed.

KEYWORDS: Cor triatriatum dexter, echocardiography.

INTRODUCTION: Cor triatriatum dexter, or partitioning of the right atrium (RA) to form a triatrial heart, is an extremely rare congenital anomaly that is caused by the persistence of the right valve of the sinus venosus.[1] The incidence of cor triatriatum is approximately $0.1 \%$ of congenital heart malformation. ${ }^{[2]}$ Typically, the right atrial partition is due to exaggerated fetal eustachian and thebesian valves, which together form an incomplete septum across the lower part of the atrium. This septum may range from a reticulum to a substantial sheet of tissue.[1]

CASE REPORT: A 45-year-old female was admitted to the gynaecology ward for undergoing surgery of an ovarian tumour. Patient was sent for routine pre-surgery cardiological evaluation in view of effort intolerance and clinical examination including the cardiovascular system examination was unremarkable. Transthoracic echocardiography (TTE) showed partitioned right atrium by a membrane which was nonrestrictive allowing passage of blood from superior to inferior chamber evidenced by the colour doppler and pulse wave doppler. There was no associated atrial septal defect or obstruction to right ventricular inflow. In view of nonrestrictive membrane patient was managed conservatively.

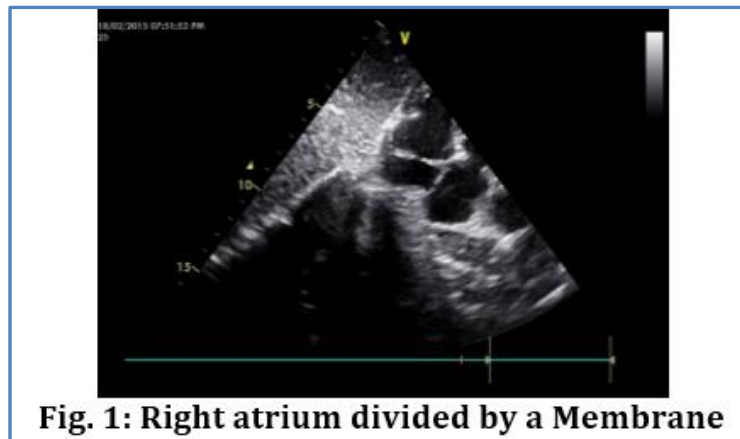

Fig. 1: Right atrium divided by a Membrane

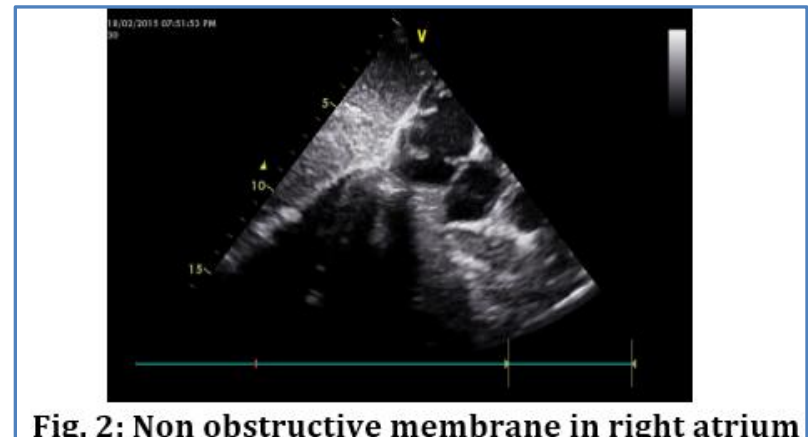

Fig. 2: Non obstructive membrane in right atrium 


\section{CASE REPORT}



Fig. 3: Flow through the membrane

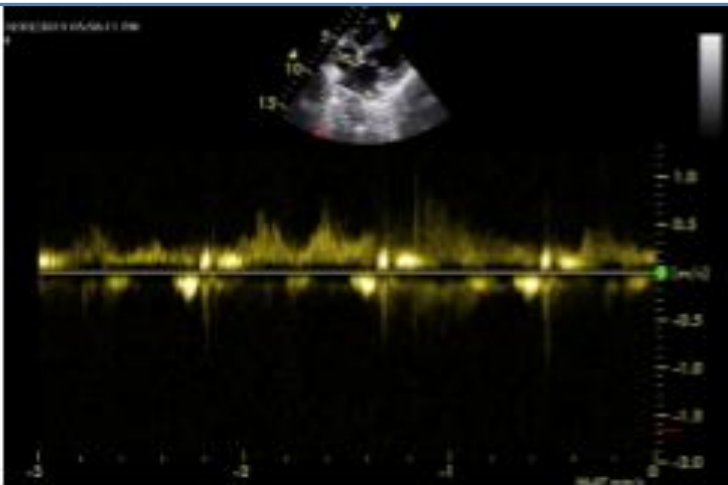

Fig. 4: Non obstructive membrane in pulse wave Doppler

DISCUSSION: During embryogenesis, the right horn of the sinus venosus incorporates into the right atrium to form the smooth posterior portion of the right atrium, whereas the original embryologic right atrium forms the trabeculated anterior portion. The connection between the right horn of the sinus venosus and the embryologic right atrium is the sinoatrial orifice, which is flanked on either side by two valvular folds, the right and left venous valves. During this incorporation at some point, the right valve of the right horn of the sinus venosus divides the right atrium into two. Normally, the valve regresses by approximately 12 weeks gestation and leaves behind the crista terminalis superiorly and the eustachian valve of the inferior vena cava and the thebesian valve of the coronary sinus inferiorly. Complete persistence of the right sinus valve results in a membrane separating the smooth and trabeculated portions of the right atrium, constituting cor triatriatum dexter.[3]

Cor triatriatum dexter has varying clinical manifestations depending on the degree of partitioning or septation of the right atrium. When the septation is mild, the condition is often asymptomatic and is an incidental finding during surgery to correct other cardiac abnormalities or during echocardiography as represented in our case. More severe septation can cause right-sided heart failure and elevated central venous pressures due to obstruction of the tricuspid valve, the right ventricular outflow tract, or the inferior vena cava. ${ }^{[1]}$ Cor triatriatum dexter can occur as an isolated cardiac anomaly or may be associated with pulmonary artery stenosis or atresia, tricuspid valve abnormality, ASD, and Ebstein anomaly. ${ }^{[4]}$ Cyanosis is a very rare presentation as represented by Hoye and colleagues in a neonate with cor triatriatum dexter with cyanosis having significant obstruction to right ventricular inflow. Most of the flow was directed to the left atrium through a patent foramen ovale.[5]

Since many patients are asymptomatic, the diagnosis of cor triatriatum dexter often is determined at postmortem examination. Antemortem diagnosis can be done by echocardiography or MRI. There have been numerous reports about the use of echocardiography to determine the diagnosis noninvasively from mid-1980s to early 1990s.[6,7] MRI has higher detection rate of cor triatrium dexter as compared to conventional echocardiography.[8] Baweja et al., reported a case in which 3D transesophageal echocardiography was able to detect cor triatriatum dexter in partial atrioventricularseptal defect (AVSD) patient that was missed by 2D echocardiography. ${ }^{[9]}$ Asymptomatic patients are generally not treated unless they are undergoing cardiac surgery for other reasons. In the past, the mainstay of treatment for symptomatic patients has been surgical 
resection of the dividing membrane. ${ }^{[2-4]}$ Recently, percutaneous catheter disruption of the membrane has been reported and has been suggested as a preferred alternative to open heart surgery.[10]

CONCLUSION: We hereby report a rare case of Cor triatrium Dexter non obstructive in nature although in restrictive cases it requires an early intervention. Clinically very difficult to distinguish, simple transthoracic echocardiography can delineate those lesions. During echocardiography one should not forget to rule out associated ASD and pulmonary stenosis. Although routine presurgery echo is not always warranted, one should be vigilant enough not to miss those rare anomalies.

\section{REFERENCES:}

1. Embrey RP. Cor triatriatum, pulmonary vein stenosis, atresia of the common pulmonary vein. In: Mavroudis C, Backer CL, editors. Pediatric cardiac surgery. 2nd ed. St Louis: Mosby-Year Book; 1994. pp. 503-4.

2. Arrants JE, Riopel DA, Catalano PW. Cor triatriatum: Preoperative diagnosis and successful surgical correction in a ten-week-old infant. Chest. 1973; 63: 1027-8.

3. Ott DA, Cooley DA, Angelini P, Leachman RD. Successful surgical correction of symptomatic cor triatriatum dexter. J Thorac Cardiovasc Surg. 1979; 78: 573-5.

4. Mazzucco A, Bortolotti U, Gallucci V, Del Torso S, Pellegrino P. Successful repair of symptomatic cor triatriatum dexter in infancy. J Thorac Cardiovasc Surg. 1983; 85: 140-3.

5. Hoye DJ, Wilson EC, Fyfe DA, Guzzetta NA. Cor triatriatum dexter: A rare cause of neonatal cyanosis. Anesth Analg. 2010; 110: 716-8

6. Burton DA, Chin A, Weinber PM, Pigott JD. Identification of cor triatriatum dexter by twodimensional echocardiography. Am J Cardiol. 1987; 60: 409-10.

7. Fiorilli R, Argento G, Tomasco B, Serino W. Cor triatriatum dexter diagnosed by transesophageal echocardiography. J Clin Ultrasound. 1995; 23: 502-4.

8. Masui T, Seelos KC, Kersting-Sommerhoff BA, Higgins CB. Abnormalities of the pulmonary veins: Evaluation with MR imaging and comparison with cardiac angiography and echocardiography. Radiology. 1991; 181: 645-9.

9. Baweja G, Nanda NC, Kirklin JK. Definitive diagnosis of cor triatriatum with common atrium by three-dimensional transesophageal echocardiography in an adult. Echocardiography. 2004; 21: 303-6.

10. Savas V, Samyn J, Schreiber TL, Hauser A, O’Neill WW. Cor triatriatum dexter: Recognition and percutaneous transluminal correction. Cathet Cardiovascular Diagn. 1991; 23: 183-6. 


\section{CASE REPORT}

\section{AUTHORS:}

1. Debasish Das

2. Satyabrata Guru

\section{PARTICULARS OF CONTRIBUTORS:}

1. Assistant Professor, Department of Cardiology, AIMS, Bhubaneswar.

2. Senior Resident, Department of Medicine, AIMS, Bhubaneswar.

FINANCIAL OR OTHER COMPETING INTERESTS: None

\section{NAME ADDRESS EMAIL ID OF THE} CORRESPONDING AUTHOR:

Dr. Debasish Das, Assistant Professor, Department of Cardiology, AIMS, Bhubaneswar.

E-mail: dasdebasish54@gmail.com

Date of Submission: 27/09/2015.

Date of Peer Review: 28/09/2015. Date of Acceptance: 29/09/2015. Date of Publishing: 01/10/2015. 